\title{
Documents et notes sur les écrits de chirurgie militaire et sur les activités en Italie du médecin genevois Louis Appia
}

Par Bruno Zanobio

A propos de la réalisation de la Croix-Rouge, il me semble que les études historiques se sont polarisées d'une façon prédominante sur l'importance novatrice des contenus juridiques, économiques, sociaux, etc., de la bienfaisante institution, tandis que d'autres thèmes techniques, notamment dans le domaine sanitaire et médicochirurgical, ont été moins souvent considérés; et ceci, aussi bien en ce qui concerne les événements que les personnes.

Par exemple, nous connaissons assez peu le rôle joué par les médecins dans la préparation de la Conférence de Genève. Dans cette optique, il m'a semblé justifié d'effectuer une étude sur Louis Appia (Hanau 1818-Genève 1898), dans le but de connaître ses écrits de chirurgie militaire et de retrouver les traces de sa présence, directe et indirecte, en Italie, de façon à pouvoir contribuer à mieux dessiner sa physionomie d'homme et de chirurgien. Il m'a semblé en outre qu'une étude de ce genre pouvait exprimer la sympathie de l'ancienne Faculté Médicale de l'Alma Mater Ticinensis à laquelle j'ai l'honneur d'appartenir, pour sa consœur genevoise, à l'occasion de son premier Centenaire.

Il n'existe pratiquement aucune étude systématique sur Appia, hormis celle (Genève/Paris 1959) de Roger Boppe, étude qui, entre autres, pourrait échapper aux spécialistes, étant donné qu'elle porte comme titre principal L'homme et la guerre, alors que l'on apprend seulement par le sous-titre qu'elle concerne en réalité Le docteur Louis Appia et les débuts de la Croix-Rouge. D'autre part, cet ouvrage, de par ses caractéristiques et son organisation méthodologique, ne se propose pas de satisfaire les exigences de connaissance de l'historien de la médecine.

L'étude que je suis en train de poursuivre s'effectue à travers l'examen de sources bibliographiques; la consultation d'ouvrages, de journaux et de revues de l'époque; la lecture d'écrits d'Appia, publiés ou inédits ; des recherches d'archives dans différents secteurs. Je tiens à remercier sincèrement toutes les personnes et les Organismes avec qui je me suis mis en contact ou qui ont mis à ma disposition des copies de documents en leur possession; personnes et Organismes que je ne manquerai pas de citer dans l'ouvrage en entier que j'ai l'intention de publier.

A propos de la première publication d'Appia, Le Chirurgien à l'ambulance ou quelques études pratiques sur les plaies par armes à feu, suivies de lettres à un 


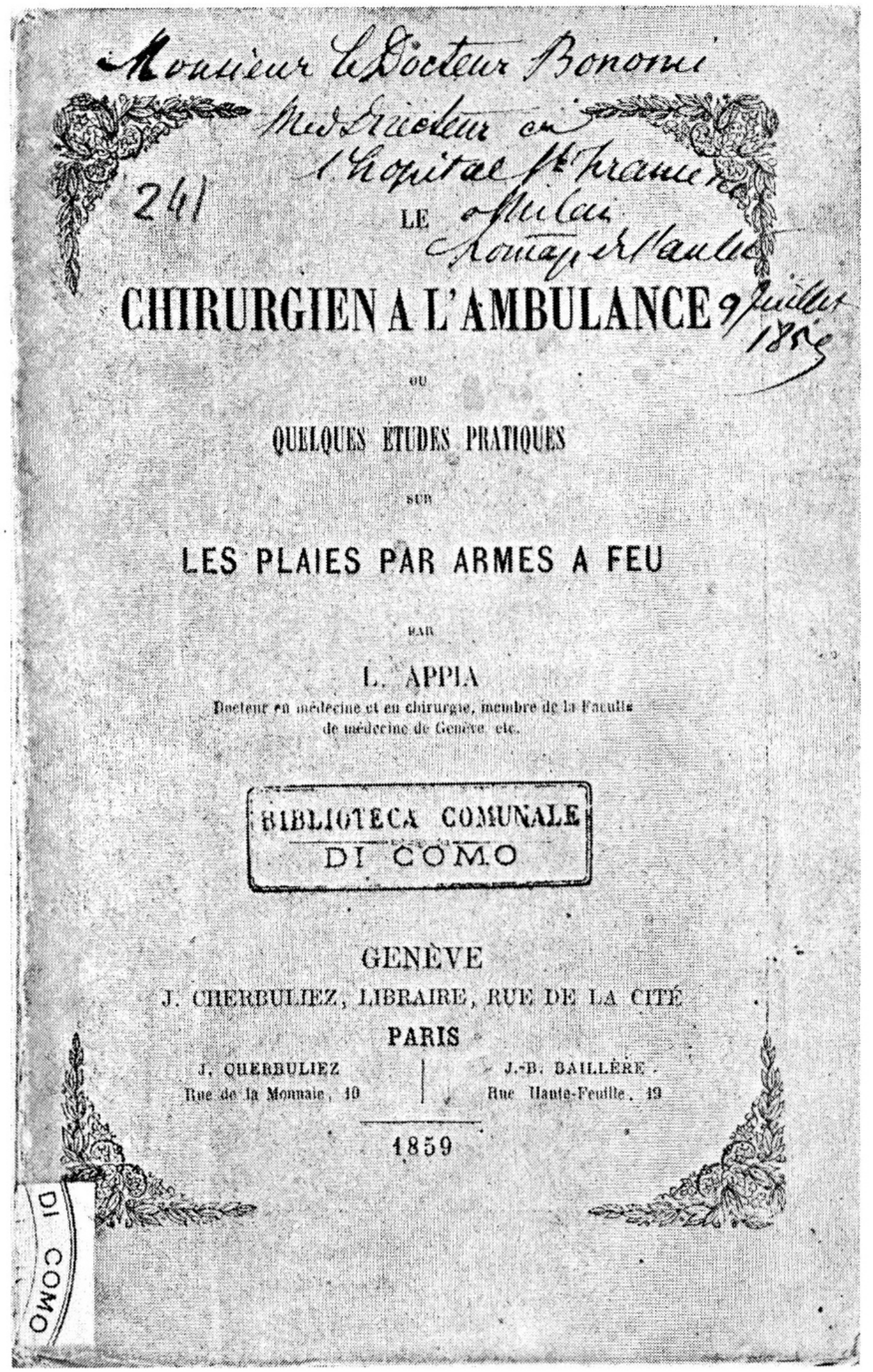




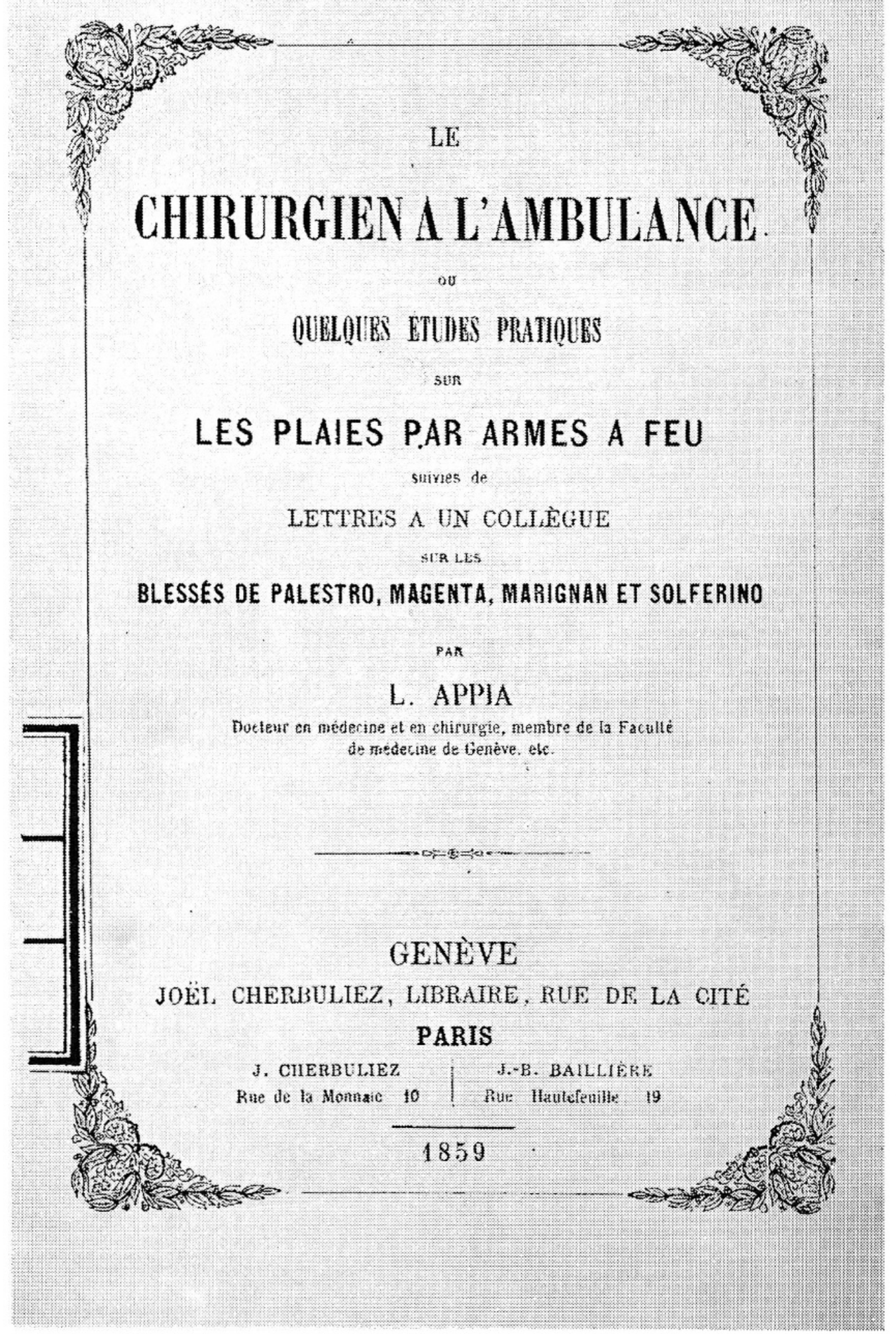


collègue sur les blessés de Palestro, Magenta, Marignan et Solferino (Genève/Paris 1859), j'ai pu en retrouver, dans la bibliothèque communale de Côme, un exemplaire, où manquent encore les Lettres à un collègue [...] et qui porte la dédicace autographe d'Appia, datée de «Milan, 9 Juillet 1859», au docteur Bonomi, indiqué comme Directeur de l'Hôpital San Francesco où étaient hospitalisés les blessés autrichiens des récentes batailles. Il s'agit évidemment d'un des rares exemplaires qu'Appia avait apportés avec lui de Genève, à l'occasion de sa première présence en Italie pour participer, dans des buts d'assistance, à la seconde guerre d'indépendance italienne.

L'esprit qui animait Appia à cette occasion ressort clairement de l'inscription gravée sur la plaque posée, tout près du palais de l'Athénée, dans la Chapelle de l'Oratoire, et que je transcris en signe d'hommage :

\author{
LE 29 JUIN 1859, QUAND SE TROUVAIT \\ ASSEMBLEE, DANS CETTE CHAPELLE, \\ LA SOCIÉTÉ ÉVANGÉLIQUE DE GENÈVE, \\ LE PROFESSEUR J.-H.MERLE D'AUBIGNE, \\ REPRENANT LE VEU DU DR. LOUIS APPIA, \\ ADRESSA AUX CHRÉTIENS, FILS DU RÉVEIL, \\ UN APPEL SOLENNEL AUQUEL RÉPONDIRENT: \\ CHARPIOT. ESTRABAND. KOEVNE. WAUTHIER. \\ ÉTUDIANTS FRANÇAIS ET BELGES À LA \\ FACULTÉ DE THÉOLOGIE DE L'ORATOIRE \\ PREMIËRE MISSION INTERNATIONALE \\ DE SECOURS AUX BLESSÉS \\ SUR LES CHAMPS DE BATAILLE DE LOMBARDIE; \\ HUMBLE PRÉLUDE DE L'CEUVRE DE LA \\ CROIX گ ROUGE \\ FONDEE EN OCTOBRE 1863 \\ «QU'ON RENDE GLOIRE À L'ÉTERNEL»ÉSAÏE. XLII. 12
}

Je suis en train de faire des recherches pour essayer de connaître, si possible, les rapports qui existaient entre Appia et Bonomi. J'ai examiné Le chirurgien à l'ambulance [...] également sous l'aspect technique et $\mathrm{j}$ 'ai pu me rendre compte dans quelle mesure Appia était au courant des problèmes médico-chirurgicaux débattus à l'époque. J'ai également utilisé à ce sujet, l'ouvrage I feriti del 1859 rédigé il y a quelque temps par notre Groupe d'étude sous la direction de Luigi Belloni ${ }^{1}$.

Le concours lancé en 1861 par l'Académie Pontaniana de Naples, sous les auspices et avec l'aide financière de Ferdinando Palasciano (1815-1891), pour 
attribuer un prix à 100 aphorismes sur le traitement des blessures provoquées par des armes à feu, subit de nombreuses vicissitudes, ainsi que j'ai pu le constater d'après une étude systématique des actes de l'Académie.

Le concours se termina finalement par le partage du prix $(2 / 3+1 / 3)$ entre deux concurrents, l'un desquels fut justement Appia. Des 108 aphorismes qu'il avait présentés, la Commission jugea digne du prix seulement les 18 sur les Règles pour le transport des blessés, qui furent les seuls à être publiés dans le Manuale di chirurgia militare, édité en 1862 par l'Académie Pontaniana. Autour de ce petit manuel se développèrent bientôt des polémiques sérieuses qui impliquèrent, directement ou indirectement, à travers l'opposition des milieux piémontais et des milieux napolitains, l'Académie Pontaniana, Palasciano, un des concurrents qui n'avait pas reçu de prix (Maturino De Sanctis), les Ministres de l'Instruction Publique, de la Guerre et de la Marine, ainsi que Francesco Cortese, Inspecteur Sanitaire de l'Armée italienne, laquelle toutefois, avait gardé jusqu'à une date récente le nom d'Armée "piémontaise».

Cortese publie en 1862/63 sa Guida teorico-pratica del medico militare in Campagna; toutefois, il n'y cite pas les écrits chirurgicaux d'Appia, bien qu'il ait dû certainement le connaître, soit à la suite des polémiques que je viens de rappeler, soit parce qu'Appia avait été décoré, dès 1859, par la Maison de Savoie, de l'Ordre des Saints Maurice et Lazare auquel appartenait Cortese lui-même.

Par contre, Appia est cité par le bernois Hermann Demme dans le premier volume Allgemeine Chirurgie der Kriegswunden. Nach Erfahrungen in den Norditalienischen Hospitälern von 1859 de ses Militär-Chirurgische Studien in den Italienischen Lazarethen von 1859 (Würzburg 1861).

Je me suis mis en rapport avec l'Académie Pontaniana, dans l'espoir de retrouver une éventuelle documentation sur Appia, mais on m'a exposé de grosses difficultés, étant donné l'état lacunaire des archives, dû aux dégâts provoqués par la dernière guerre. Je compte effectuer néanmoins des recherches dans les Archives d'Etat de Naples : il serait en effet intéressant pouvoir établir quels rapports éventuels existaient entre Appia et Palasciano.

La partie des aphorismes d'Appia qui n'avait pas été publiée à l'époque et qui est restée inédite jusqu'ici, est conservée au Musée d'Histoire des Sciences de Genève, qui m'en a aimablement procuré des photocopies. Je reporte ici, à titre d'exemple, en format réduit, une page de ces manuscrits que je suis en train de transcrire pour leur publication ultérieure. On pourra ainsi comparer ces écrits d'Appia avec ceux de ses prédécesseurs et de ses contemporains (Armand, Baudens, Larrey, etc.), ainsi qu'avec ceux des auteurs qui suivirent immédiatement (Arrault, Baroffio, Cortese, De Sanctis, Legouest, etc.). 
En gémeral le thirugies ne so preoveriperas

hew outre mesure sle ba balle restec deus

La plaic, quant elle ett nacceftille ò thi:

itrument on an daigh. - laxut cle tenter thectraction de la balles pas

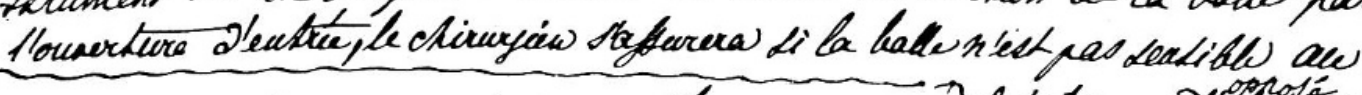

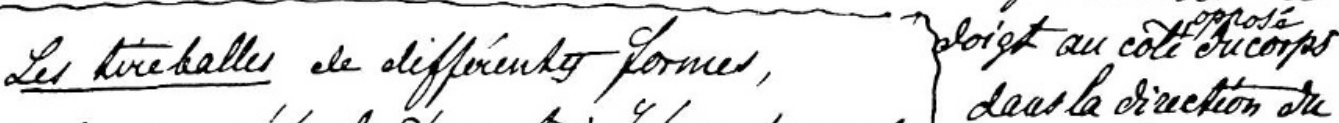

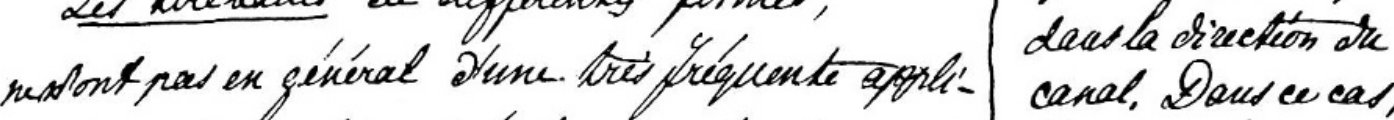
- cotion Lextraction sécéche daus le pliuspene

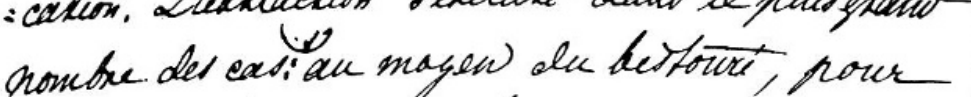

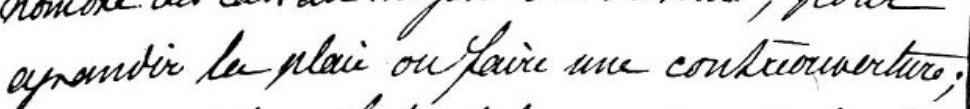

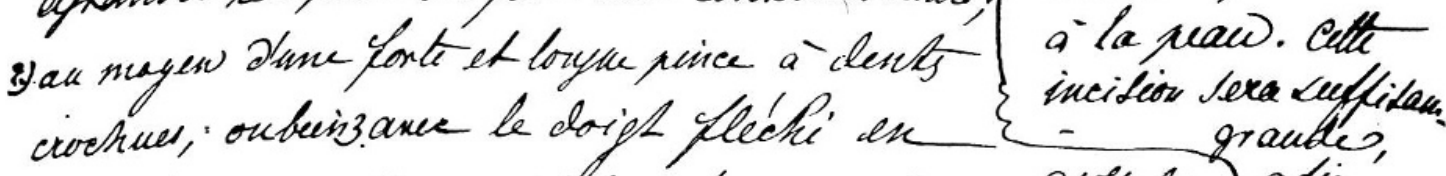

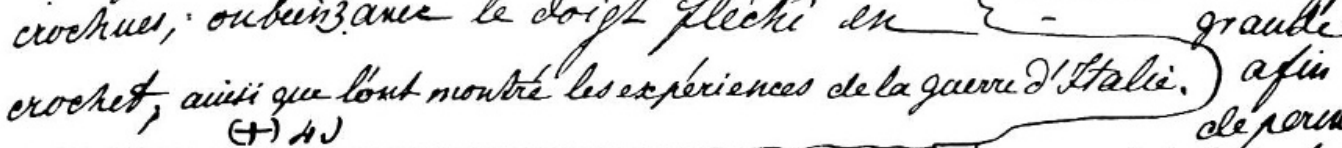
"in - Lión de feraraumagen D'une suciple incitiós a'la neaw. cette incition Sera culfitan.

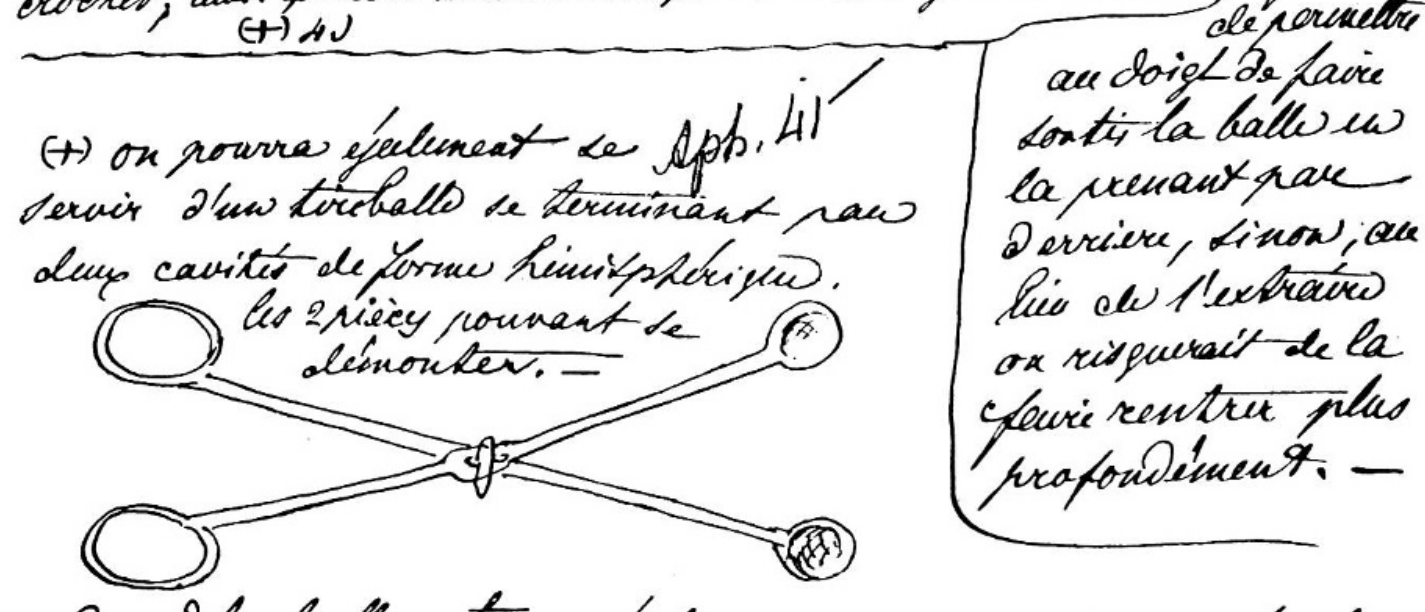

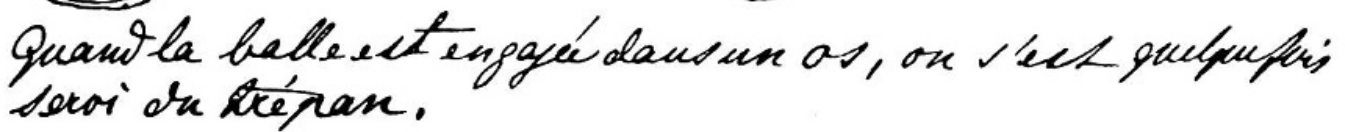

Il n'est pas exclus qu'à travers une revue attentive des écrits chirurgicaux de l'époque, nous puissions trouver quelques auteurs qui rappellent Appia. Je cite, par exemple, la Memoria sulle amputazioni... (Turin 1862) du «médecin de bataillon» Cesare Lombroso (1836-1909), publiée dans le Giornale di Medicina Militare. 
Appia participa en outre, dans des buts d'assistance, à la bataille de Bezzecca et à la Campagne du Tyrol de 1866, au cours de laquelle il rencontra Garibaldi. A l'aide des deux volumes sur Georges Appia, Pasteur et Professeur en Italie et à Paris, imprimés il y a plusieurs années par Flammarion (Paris, s. d.), et de la toute récente étude (Bollettino della Società di Studi Valdesi, 1976) du Père Dominicain Paolo Gnudi sur les Valdesi nella terza guerra d'Indipendenza (1866), j'essaie de définir l'état du problème de l'assistance aux blessés à cette époque et la part qui revient à Appia dans la solution de ce problème. Il ne faut pas oublier qu'à cette époque Appia n'était pas en bonne santé et que sa décision de retourner en Italie pour une autre expérience d'assistance en guerre (il venait de rentrer de celle du Schleswig, 1864) fut certainement due, en grande partie, à l'influence de son frère plus jeune, le pasteur vaudois Georges Appia (1827-1910), un personnage marquant.

Sur sa présence en Italie à cette occasion, Appia nous a laissé deux petits ouvrages dont l'un, Les blessés de la bataille de Bezzecca dans la vallée de Tiarno 21 Juillet 1866 (Genève 1866) a été traduit en italien et publié il y a quelques années par Antonio Fappani.

A travers la lecture des journaux médicaux et des documents de l'époque, je suis en train de reconstruire les motifs des polémiques à propos de l'organisation des services sanitaires militaires et de l'assistance aux blessés, polémiques qui étaient déjà vives pendant la campagne de 1866 et qui continuèrent même après.

Aucune allusion à la présence d'Appia ne se trouve dans l'ouvrage de Cortese intitulé Della campagna combattuta dalle armi italiane nell'anno 1866 risguardante lo stato sanitario dell'esercito et publié dans le vol. XII des Atti del R. Istituto veneto di scienze, lettere ed arti.

J'ai pu retrouver le diplôme par lequel, après la bataille de Bezzecca, Appia avait été promu de Chevalier à Officier de l'Ordre des Saints Maurice et Lazare. Etant donné qu'il ressort de ce diplôme que la proposition pour la promotion était partie du Ministre des Affaires Etrangères, je me suis mis en contact avec les Archives de ce Ministère pour essayer de retrouver le dossier qui s'y rapporte. Ce dernier pourrait en effet nous faire connaître d'une façon détaillée la mention de la décoration et présenter, par conséquent, un certain intérêt.

A Genève, l'on a conservé un brassard blanc, marqué de la croix rouge, que la tradition indique comme étant celui qu'Appia portait dans le Schleswig (1864), à Bezzecca (1866) et ensuite en France (1870). J'ai trouvé au Musée Vaudois de Torre Pellice un brassard identique à celui de Genève; il a été donné au Musée par Madame Béatrice Appia Blacher, domiciliée actuellement à Paris, comme étant un brassard qui a sûrement appartenu à Louis Appia. 


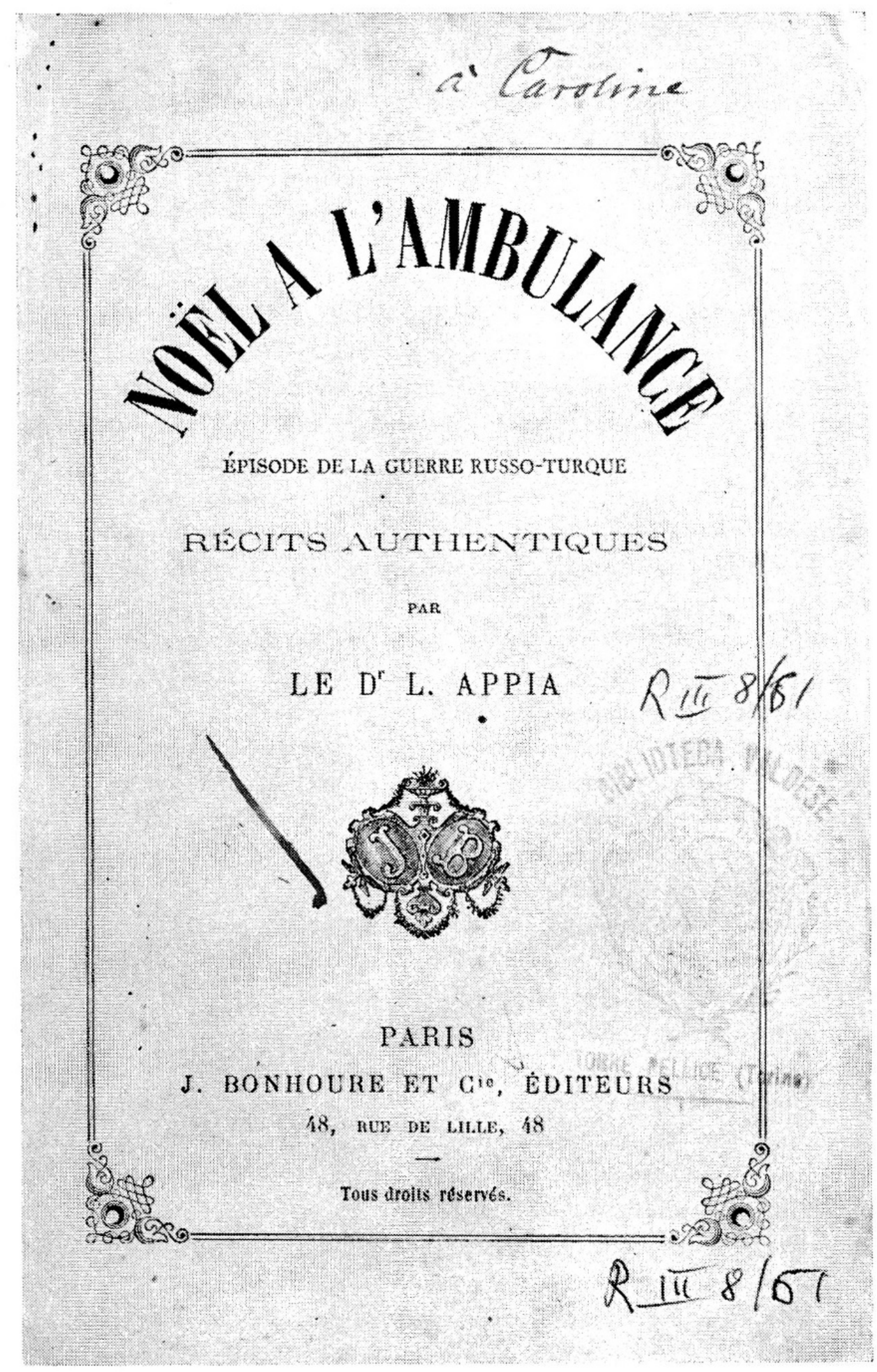


Rappelons incidemment que, parmi ceux qui participèrent aux guerres de 1866 et de 1870 avec des "patrouilles» de secours ou des «ambulances sanitaires civiles», on compte également le fameux anatomiste de Turin, Carlo Giacomini ${ }^{2}$ (1840-1898) qui fut certainement un des premiers à utiliser le brassard blanc avec la croix rouge. La piste de Giacomini pourrait, elle aussi, se révéler intéressante pour une meilleure connaissance d'Appia.

En ce qui concerne la brochure Noël à l'ambulance [...] (Paris s.d.) sur la guerre russo-turque de 1881, je signale que, dans la bibliothèque vaudoise de Torre Pellice, j'en ai trouvé un exemplaire avec la dédicace «à Caroline» qui semble autographe et qui fait penser à un hommage d'Appia à sa femme.

La dernière présence connue d'Appia en Italie fut en 1892, lorsqu'il intervint, à Rome, à la $\mathrm{V}^{\mathrm{e}}$ Conférence Internationale de la Croix-Rouge, en tant que membre du Comité International. On ne peut pas exclure que, après un réexamen attentif des actes de la Conférence, et en lisant entre les lignes, on puisse apprendre sur Appia quelque chose de plus de ce qui apparaît dans ses interventions officielles et dans les documents, bien qu'intéressants, jusqu'ici trouvés à ma demande, dans les Archives Centrales de l'Etat et provenant des Archives du Palais du Quirinal.

Dans le cadre de mes recherches sur Appia, j'ai également en cours des contacts avec l'Académie des Sciences de l'Institut de France, de même que je suis en train de consulter de façon systématique le Giornale di Medicina Militare, la Gazzetta Medica Lombarda, les Annali Universali di Medicina e Chirurgia, ainsi que d'autres revues de l'époque qui se sont toutefois révélées, jusqu'ici, assez pauvres en notices; résultat sur le sens duquel il m'est encore difficile de me prononcer. J'estime par contre, sur la base des données déjà connues et de celles récemment acquises - et qui sont susceptibles d'être encore complétées - que la personnalité d'Appia, aussi bien comme homme que comme chirurgien, est en train de révéler des aspects d'une certaine complexité et présentant un intérêt indéniable.

\section{Notes}

${ }^{1}$ Le 31 mai 1959, nous avons tenu à Milan, dans les locaux de l'ancien hôpital militaire de Saint-Ambroise (siège actuel de l'Université Catholique du S. Cœur), une réunion d'études consacrée à I feriti del 1859, dont les Actes ont paru dans Minerva Medica 51 (1960), p.1393-1430 (suppl. au n. 31 du 18 Avril 1960), avec le sommaire suivant: L. Belloni, Introduzione; A. Carimati, Innovazioni balistiche e ripercussioni traumatologiche. Organizzazione sanitaria militare; P.F.Peregalli, Problemi chirurgici della campagna del 1859 ; 
B.Zanobio, Profilassi delle infezioni delle ferite; E.Poli, Il primo tentativo di terapia curarica del tetano; F. Grondona, Gli Ospedali Militari di Milano e di Brescia nel 1859 ; V. Ghetti, Nascita della Croce Rossa; L.Belloni, A.Carimati e F.Grondona, Spunti di patologia all'Ossario di Solferino.

Cf. inoltre: L. Belloni, Un ricordo di Solferino, in: Symposium Ciba 7 (1959), p.41-44; B.Zanobio, Il sistema della dispersione dei malati («Kranken-Zerstreuungs-System») di Felix von Kraus nella campagna d'Italia del 1859, in: Rendiconti d. Istit. Lombardo (Classe di Scienze) 95 (1961), p.135-150.

2 F. Loreti, Contributo alla storia dello «Studio » anatomico della Università di Torino. Carlo Giacomini (1840-1898), in : Memorie dell'Accademia delle Scienze di Torino. Cl. di Sc. Fis., Mat. e Nat., Serie $4^{\circ}$ (1963), n. 2.

\section{Summary}

Bio-bibliographical details on Louis Appia's activities during the wars of independence in Italy. The sources of further work on the subject are indicated.

Prof. Dr. med. Bruno Zanobio

Cattedra di Storia della Medicina

Museo per la Storia della Università di Pavia

Strada Nuova, 65

I-27100 Pavia 